\title{
Atraumatic Extensor Hallucis Longus Rupture
}

\author{
Gennady Kolodenker ${ }^{1 *}$, Michael Heaslet ${ }^{1}$ and David Yang ${ }^{2}$ \\ ${ }^{1}$ Doctor of Podiatric Medicine, Foot and Ankle Specialist, Irvine, California, USA \\ ${ }^{2}$ Doctor of Podiatric Medicine, Newport Foot and Ankle Center, Irvine, USA
}

Received: March 19, 2014; Accepted: May 15, 2014; Published: May 20, 2014

*Corresponding author: Gennady Kolodenker, DPM, AACFAS, Foot and Ankle Specialist, 4950 Barranca Parkway, Suite 308, Irvine, CA 92604, USA. Tel: (949) 651-1202; Fax: (949) 552-9493; Email: www.OCPodiatry.com

\begin{abstract}
This article is a case presentation of a rare finding in tendon injuries. Atraumatic extensor hallucis longus tendon rupture with surgical repair and recovery. Patients do well with primary repair of the tendon.
\end{abstract}

Keywords: Atraumatic Extensor Hallucis Longus Rupture; Spontaneous Tear

\section{Introduction}

Spontaneous atraumatic tendon ruptures are a rare entity [1]. The vast majority of reported extensor hallucis longus (EHL) tendon injuries documented in the literature have been secondary to a laceration [2-6]. Extensor injuries account for 1.58 to 1.68 percent of all tendon injuries $[3,7]$. This is the very first case reported of an atraumatic EHL tendon tear of a patient with no prior medical problems, steroid use or injection.

\section{Case Report}

This is a 69 year old female with no prior medical problems. The patient presented complaining of pain in her left foot. A few days prior to presentation she was walking on the boardwalk with her husband. Patient was wearing her regular sneaker. She has done this walk many times before without any incident. A few steps into the walk the patient noted a sharp pain at the dorsum of her foot. She attempted to keep walking, but could not figure out why her foot has become swollen and ecchymotic. Patient denies taking any medication. Specifically patient denies taking any antibiotics, steroids, or receiving injections. She has a surgical history significant for cholecystectomy at age thirty nine and an appendectomy at the age of ten. Two years after the injury patient is doing well with full range of motion at the $1^{\text {st }}$ metatarsophalangeal joint.

At initial evaluation it was noted that there is a dorsal palpable dell over the central area of the first metatarsal. The dell is easily visualized on the x-ray of her left foot. Secondary to the rarity of this presentation an MRI was ordered to confirm the diagnosis.

The MRI revealed a complete rupture of the extensor hallucis longus tendon at the level of the mid metatarsal. There is a
$4.8 \mathrm{~cm}$ proximal retraction of the tendon. Tenosynovitis of the remainder of the tendon is present. Another incidental finding was osteoarthritis of the first metatarsophalangeal joint. In addition, periarticular bony erosions are present. Additional periarticular bony erosions are seen around the third through fifth tarsometatrsal joint. These findings raise the possibility of superimposed gout.

After confirmation of EHL rupture, surgical correction was elected.

The patient was placed on the operating room table in a supine position. Hemostastis was achieved with a well padded thigh tourniquet at $300 \mathrm{mmHg}$. The left lower extremity was prepped and draped in a normal sterile fashion. A linear incision was made from the first metatarsophalangeal joint to the ankle joint along the path of the EHL tendon. The synovial sheath was noted to be intact with central areas of rupture. A complete rupture of the EHL tendon was noted with a gap of about 7 centimeters. The extensor capsularis tendon was visualized and noted to be ruptured distally near the insertion with minimal retraction. The remainder of the capsularis tendon was noted to be intact without any tendonopathy.

The distal and proximal ends of EHL tendon were identified. Approximately $2 \mathrm{~mm}$ of tendon ends were sharply excised and sent to pathology for further evaluation. Unfortunately the specimen was lost and no microscopic evaluation was available. The excised tendon felt calific upon palpation.

The ruptured extensor hallucis longus tendon was repaired utilizing a 2-0 fiberwire suture in a Krakow fashion. After repair, it was noted that there was increased extensory force on the hallux with a taught EHL. A z-lengthening of the proximal healthy aspect of the EHL tendon was performed and secured with 2-0 fiberwire.

The extensor capsularis tendon was utilized to reinforce the EHL tendon. The capsularis tendon was sutured upon the EHL tendon utilizing 2-0 fiberwire. The surgical site was irrigated and closed in layers. Care was taken to suture together the synovial sheath with 2-0 vicryl. Patient was placed in a posterior splint after the surgical correction. 
Patient was placed in a walking boot seventeen days after the surgical procedure.

\section{Discussion}

Most reported cases of spontaneous tendon ruptures have a patient history significant for systemic disease, such as rheumatoid arthritis, chronic steroid use, and/or injection of steroids to the tendon [7-9]. Loss of extensor function will decrease ankle strength [10]. The patient presented in the article has no history of systemic disease, steroids use, or surgical treatment. There seems to be a common theme in the literature of injectable or oral steroids weakening tendons $[8,9,11]$.

Since the specimen was lost, no pathology report is available. A 20 year study by Kannus of almost 900 spontaneous ruptures confirmed that every ruptures tendon had some degree of degenerative changes. The pathologic changes can be seen as hypoxic degenerative tendinopathy, mucoid degeneration, tendolipomatosis, calcifying tendinopathy, intratendinous foreign body, rheumatoid tendonitis, a xanthoma, or an intratendinous ganglion [7]. Even without the microscopic examination, it was evident on palpation that the tendon was abnormal.

\section{Summary/Conclusion}

Even though extremely rare, it is possible to have an acute atraumatic rupture of the EHL tendon. Some more insight into tendon degeneration is needed. It is important to repair extensor hallucis longus tendon in order to gain mobility of the hallux at the first metatarsophalangeal joint. Without the function of the longus tendon patients will complain that their hallux drags on the floor. After repair and immobilization, protected weigh bearing followed by physical therapy will expedite the healing process.

\section{References}

1. Wei SY, Kneeland JB, Okereke E (1998) Complete atraumatic rupture of the flexor hallucis longus tendon: a case report and review of the literature. Foot Ankle Int 19(7): 472-474.

2. Bronner S, Ojofeitimi S, Rose D (2008) Repair and Rehabilitation of Extensor Hallucis Longus and Brevis Tendon Lacerations in a Professional Dancer. J Orthop Sports Phys Ther 38(6): 362-370.

3. Kass JC, Palumbo F, Mehl S, Camarinos N (1997) Extensor Hallucis Longus Tendon Injury: An In-depth Analysis and Treatment Protocol. J Foot Ankle Surg 36(1): 24-27.

4. Thompson FM, Snow SW, Hershon SJ (1993) Spontaneous atraumatic rupture of the flexor hallucis longus tendon under the sustentaculum tali: case report, review of the literature, and treatment options. Foot Ankle 14(7): 414-417.

5. Scaduto AA, Crachiolo A (2000) Lacerations and ruptures of the flexor or extensor hallucis longus tendons. Foot Ankle Clin 5(3): 725-736.

6. Tuncer S, Aksu N, Iskklar U (2010) Delayed Rupture of the Extensor Hallucis Longus and Extensor Digitorum Communis Tendons After Breaching the Anterior Capsule with a Radiofrequency Probe During Ankle Arthroscopy: A Case Report. J Foot Ankle Surg 49(5): 490 e1- 490 e3.

7. Kannus P, Jozsa L (1991) Histopathological changes preceding spontaneous rupture of a tendon. A controlled study of 891 patients. J Bone Joint Surg Am 73(10): 1507-1525.

8. Kotnis RA, Halstead JC, Hormbrey PJ (1999) Atraumatic bilateral Achilles tendon rupture: an association of systemic steroid treatment. J Accid Emerg Med 16(5): 378-379.

9. Peter SR, Frank JF (2001) Review of the Literature Atraumatic Bilateral Patellar Tendon Rupture : A Case Report and Review of the Literature. J Bone Joint Surg Am 83(9): 1382-1386.

10. James Sammarco V, James Sammarco G, Carlo Henning, Solomon Chaim (2009) Surgical Repair of Acute and Chronic Tibialis Anterior Tendon Ruptures. J Bone Joint Surg Am 91(2): 325-332.

11. Poggi JJ, Hall RL (1995) Acute rupture of the extensor hallucis longus tendon. Foot Ankle Int 16(1): 41-43. 